\title{
Criticality between Cortical States
}

\author{
Antonio J. Fontenele, ${ }^{1, *}$ Nivaldo A. P. de Vasconcelos, ${ }^{1,2,3,4, *}$ Thaís Feliciano, ${ }^{1}$ Leandro A. A. Aguiar, ${ }^{1,5}$ \\ Carina Soares-Cunha, ${ }^{3,4}$ Bárbara Coimbra, ${ }^{3,4}$ Leonardo Dalla Porta, ${ }^{1,6}$ Sidarta Ribeiro, ${ }^{7}$ \\ Ana João Rodrigues, ${ }^{3,4}$ Nuno Sousa, ${ }^{3,4}$ Pedro V. Carelli, ${ }^{1, \dagger}$ and Mauro Copelli, ${ }^{1, \hbar}$ \\ ${ }^{1}$ Physics Department, Federal University of Pernambuco (UFPE), Recife, PE 50670-901, Brazil \\ ${ }^{2}$ Department of Biomedical Engineering, Federal University of Pernambuco, Recife, PE 50670-901, Brazil \\ ${ }^{3}$ Life and Health Sciences Research Institute (ICVS), School of Medicine, University of Minho, Braga 4710-057, Portugal \\ ${ }^{4}$ ICVS/3Bs-PT Government Associate Laboratory, 4806-909, Braga/Guimarães, Portugal \\ ${ }^{5}$ Departamento de Morfologia e Fisiologia Animal, Universidade Federal Rural de Pernambuco (UFRPE), \\ Recife, PE 52171-900, Brazil \\ ${ }^{6}$ Systems Neuroscience, Institut dInvestigacions Biomèdiques August Pi i Sunyer (IDIBAPS), 08036, Barcelona, Spain \\ ${ }^{7}$ Brain Institute, Federal University of Rio Grande do Norte (UFRN), Natal, RN 59056-450, Brazil
}

(Received 6 November 2018; revised manuscript received 18 February 2019; published 21 May 2019)

Since the first measurements of neuronal avalanches, the critical brain hypothesis has gained traction. However, if the brain is critical, what is the phase transition? For several decades, it has been known that the cerebral cortex operates in a diversity of regimes, ranging from highly synchronous states (with higher spiking variability) to desynchronized states (with lower spiking variability). Here, using both new and publicly available data, we test independent signatures of criticality and show that a phase transition occurs in an intermediate value of spiking variability, in both anesthetized and freely moving animals. The critical exponents point to a universality class different from mean-field directed percolation. Importantly, as the cortex hovers around this critical point, the avalanche exponents follow a linear relation that encompasses previous experimental results from different setups and is reproduced by a model.

DOI: 10.1103/PhysRevLett.122.208101

It is well established that cortical activity exhibits a rich repertoire of dynamical states [1-3]. This knowledge, initially based on electroencephalographic recordings, later reached the spiking activity of large neuronal populations of both anesthetized and freely moving animals [4,5], in which the variability level has been used as a proxy of the cortical state [5-8]. However, only recently has the diversity of cortical states been systematically considered in studies of criticality $[9,10]$.

In the first results that fueled the critical brain hypothesis [11], Beggs and Plenz observed local field potential (LFP) neuronal avalanches in vitro with power-law size distributions $P(s) \sim s^{-1.5}[12]$. The exponent coincides with that of a critical branching process, which has driven significant efforts towards the idea that the brain hovers around a critical point belonging to the mean-field-directed percolation (MF-DP) universality class [13].

Findings for spiking data have, however, raised some controversy: on one hand, power-law size distributions were found during strong, slow LFP oscillations, under ketamine-xylazine [9] and isoflurane [10] anesthesia. On the other hand, long-range time correlations (another statistical signature of criticality [14]) were observed during fast LFP oscillations in freely behaving rats, but not under ketamine-xylazine anesthesia [9]. Those results lead to a conundrum, where the signatures of a critical state might be dependent on the level of synchronization, thus challenging the whole picture of directed percolation, which involves no oscillations whatsoever, and where the system goes from an absorbing to an active state.

Here we show that the signatures of criticality in vivo vary continuously across the cortical states, and that scaling relations predicted by the theory of critical phenomena can be used to determine a well-defined critical point. We quantified the variety of cortical states $[4,5]$ in terms of the coefficient of variation (CV) $[5,6]$ of the summed population activity in the primary visual cortex $(V 1)$ of urethane-anesthetized rats [8] (Fig. S1 [15]). We recorded a total of 1628 units [Fig. 1(a), Tables S1 and S2 [15] ] in deep layers of $V 1$ ( $n=8$ rats), under spontaneous activity, during long periods $(\geq 200 \mathrm{~min}$ ). We employed highcount sites silicon probes $(64 / 32$ channels, see Supplemental Material [15]) to record spiking activity of large neuronal populations. We then extended our analyses to publicly available datasets comprising $V 1$ action potentials from one sufentanil-anesthetized monkey [22] and from freely moving nonanesthetized mice [23] (see details in the Supplemental Material [15]).

We calculated $\mathrm{CV}$, which is correlated with the Fano factor (Fig. S4 [15]), within nonoverlapping $10 \mathrm{~s}$ windows. In that timescale, CV typically changes rapidly [4-6,8] [Fig. 1(b)]. Within each of these windows, for increasing 


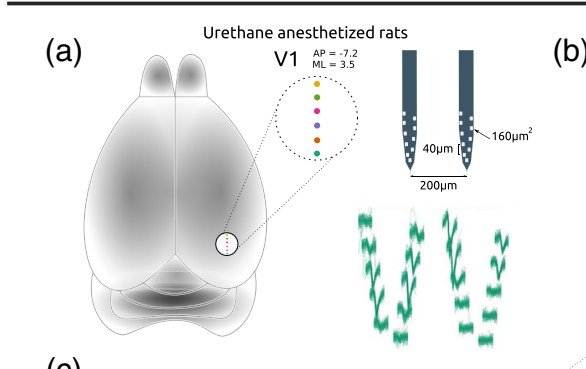

(c)

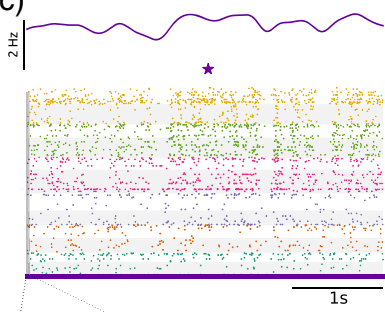

(e)

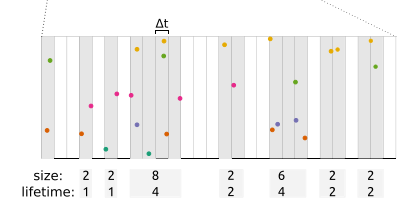

(b)

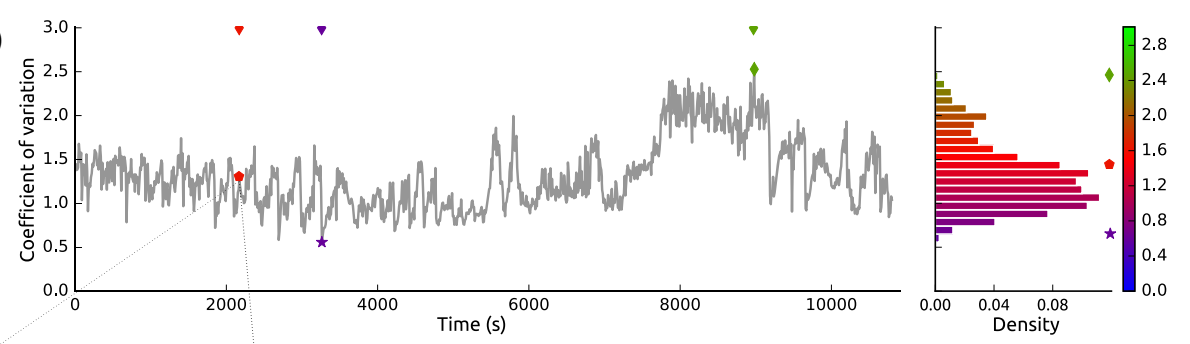

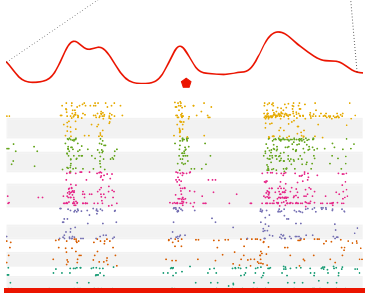

(f)

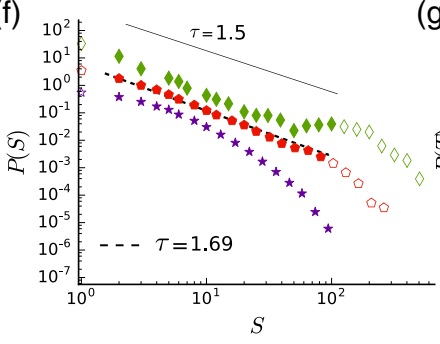

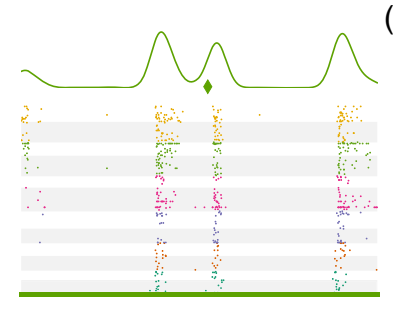

(d)

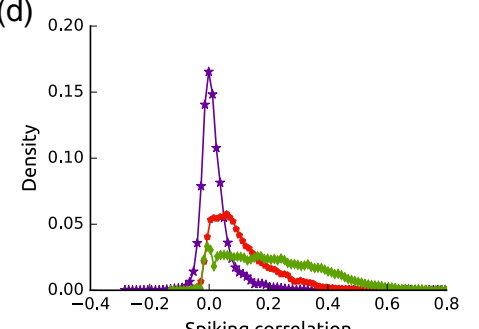

(g)

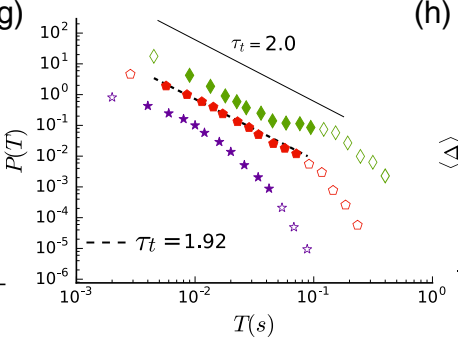

(h)

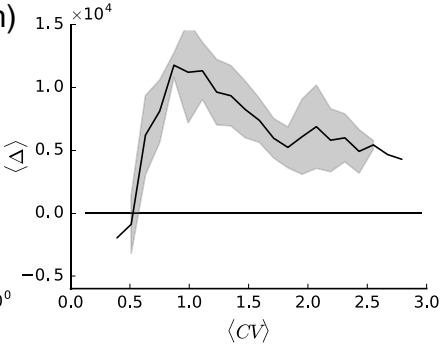

FIG. 1. Statistical properties of cortical dynamics along different levels of spiking variability. (a) Position of the six shanks (colored dots) in the primary visual cortex (V1). (Bottom right) Samples of spike waveforms. (b) (Left) Coefficient of variation (CV) of the spiking activity in $V 1$ (see also Supplemental Material, Figs. S2 and S3 [15]). Each point was calculated for a 10-s-long nonoverlapping time period; symbols (star, pentagon and diamond) indicate the level of spiking variability of three representative examples (low, intermediate, and high, respectively: $\langle\mathrm{CV}\rangle=0.38,1.48$, and 2.86). (Right) CV histogram of a single animal. The vertical color bar is used as a reference for CV scale throughout the Letter. (c) Samples of 4-s-long spiking activity across the three levels of spiking variability depicted in (b). (Top) Population rate smoothed by a Gaussian kernel $\sigma=0.1 \mathrm{~s}$. (Bottom) Raster plot: single- and multi-units (SUA = 138 and MUA = 153) with white and gray backgrounds, respectively. (d) Histogram of pairwise spiking correlation along low, intermediate, and high levels [same symbols as in (c)], where mean and standard deviation are $0.028 \pm 0.069,0.117 \pm 0.115$, and $0.224 \pm 0.165$, respectively (see Supplemental Material [15]). (e) The data are divided in nonoverlapping time bins, $\Delta t$ (see Supplemental Material [15]). Population spikes preceded and followed by silences define a spike avalanche (gray backgrounds). The number of spikes defines the avalanche size, whereas the number of bins define its lifetime. (f), (g) Distributions of size and lifetime of spiking avalanches, $P(S)$ and $P(T)$, respectively, during the dynamical states described in (d). Filled symbols indicate the range to which we fitted a power law in both cases. Solid lines show the exponents of the MF-DP universality class. (h) Relative goodness-of-fit test of the size distribution according to the Akaike Information Criterion (group data B, see Supplemental Material [15]). Positive (negative) values indicate power-law (log-normal) behavior.

values of $\mathrm{CV}$, spiking activity ranges from completely desynchronized to a strongly synchronized state [Fig. 1(c)]. In what follows, we sort results according to $\mathrm{CV}$ values and average over consecutive percentiles to obtain $\langle\mathrm{CV}\rangle$ as a representative of a given spiking variability level (Fig. S1 [15]). Different spiking correlation structures underlie those different regimes. Both mean and standard deviation of pairwise spiking correlations increase from the desynchronized state to the strongly synchronized state [Fig. 1(d), $p \ll 0.01$, rank sum test].

By dividing each $10 \mathrm{~s}$ window in short time bins ( $\Delta t \sim 2-4 \mathrm{~ms}$ ), spike avalanches are defined by the spatiotemporal spiking patterns between silent bins [Fig. 1(e)]. We used the standard definition of $\Delta t$ as the average interspike interval $[9,12]$. Since the firing rates decrease monotonously with increasing $\langle\mathrm{CV}\rangle$ [24] (Fig. S5 [15]), for each $10 \mathrm{~s}$ window a different $\Delta t$ was calculated. The size $S$ and lifetime $T$ of an avalanche are, respectively, given by the total numbers of spikes and bins within each event.

Even when bins are adjusted by firing rates, the statistics of avalanche size and lifetime differ across the range of $\langle\mathrm{CV}\rangle$ values. For instance, sampling the lower, intermediate, and upper portions of the $\langle\mathrm{CV}\rangle$ range, the degree to which the distributions of avalanche size and lifetime can be fitted by power laws (Supplemental Material [15])

$$
\begin{aligned}
& P(S) \sim S^{-\tau}, \\
& P(T) \sim T^{-\tau_{t}}
\end{aligned}
$$


varies considerably [Figs. 1(f) and $1(\mathrm{~g})]$. In particular, the exponents $\tau$ and $\tau_{t}$ do not necessarily agree with those of MF-DP. According to the Akaike criterion [Fig. 1(h), Supplemental Material [15]), power laws cease to be the best fitting distribution for sufficiently low $\langle\mathrm{CV}\rangle$ (and, if the data are shuffled, for any $\langle\mathrm{CV}\rangle$, see Figs. S6 and S7 [15]). Subsequently, we refine our analysis by checking the consistency of scaling relations across $\langle\mathrm{CV}\rangle$ values.

The theory of critical phenomena predicts that, if Eqs. (1) and (2) hold at a critical point, then so does

$$
\langle S\rangle(T) \sim T^{\frac{1}{\sigma \nu z}}
$$

where $1 /(\sigma \nu z)$ is a combination of critical exponents $[25,26]$. Our data are clearly consistent with Eq. (3), with the exponent $1 /(\sigma \nu z)$ depending on the spiking variability $\langle\mathrm{CV}\rangle$ [Fig. 2(c)]. The same exponent governs avalanche shape collapse $[25,27]$, which we also observe (Fig. S8 [15]).

(a)
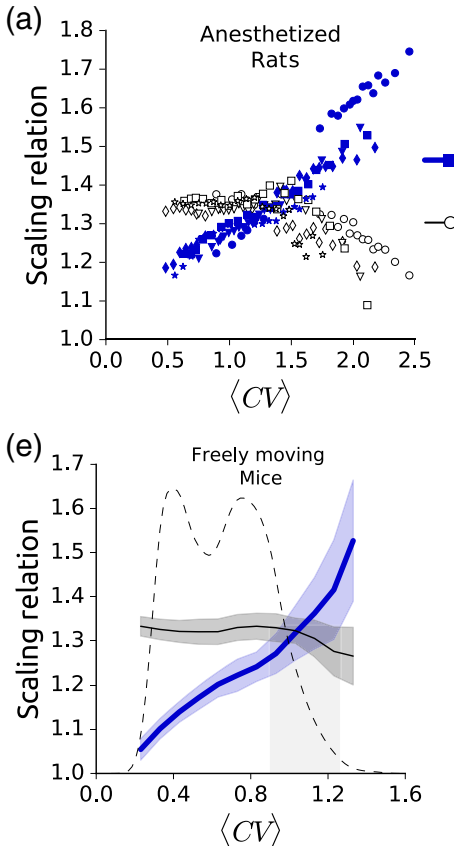

(b)

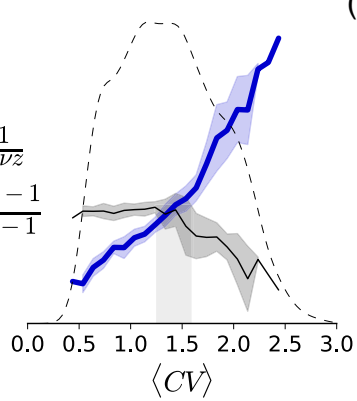

(c)

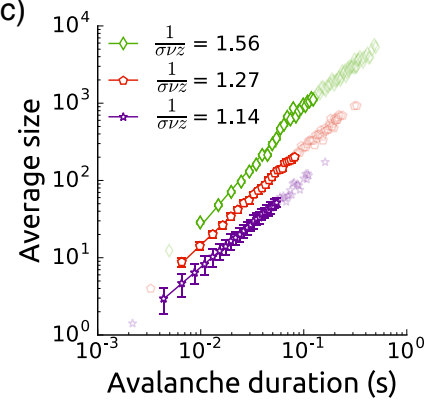

The scaling relation of Eq. (3), however, is known to hold even far from criticality [25], so it can hardly be considered a sufficient signature in itself. In fact, Touboul and Destexhe claim that Eqs. (1)-(3) can be obtained in systems that are not critical [30]. However, if the system is indeed critical, another scaling relation connecting $1 /(\sigma \nu z)$ with $\tau$ and $\tau_{t}$ is expected to hold, namely [25],

$$
\frac{\tau_{t}-1}{\tau-1}=\frac{1}{\sigma \nu z}
$$

The above relation has not been observed by Touboul and Destexhe away from criticality and is therefore considered a much stricter criterion for criticality [30]. Since both sides of the above relation can be independently evaluated, we tested whether they equal each other as the brain spontaneously traverses the different levels of spiking variability. We verified that Eq. (4) clearly holds for each animal $(n=8)$ and, strikingly, the crossing between the left and
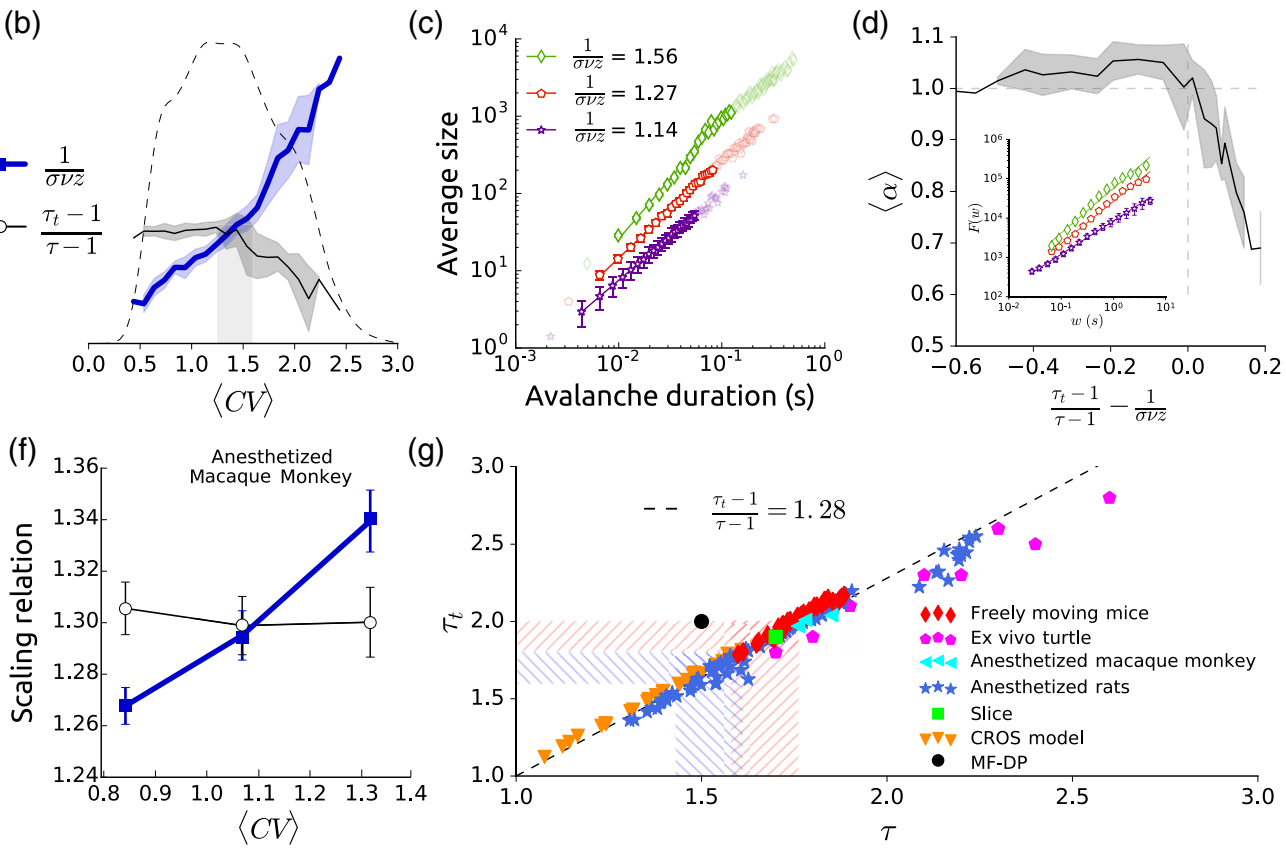

FIG. 2. Signatures of criticality as a function of the cortical spiking variability. (a) Scaling relation across the variability spectrum per animal (five urethane-anesthetized rats), where open (filled) symbols correspond to the left (right) side of Eq. (4). Each animal is represented by a different symbol type. (b) Group data for the rat experiments shown in (a). (b),(e) Solid thin (solid thick) line corresponds to the left (right) side of Eq. (4). The shadow around each curve represents its standard deviation. The dashed curve represents the relative residence time across CV values. Gray stripe in (b) represents the critical value of $\langle\mathrm{CV}\rangle_{*}=1.4 \pm 0.2$, where Eq. (4) holds, considering the standard deviations. (c) Power-law relation between size and lifetime of spike avalanches across different levels of spiking variability [same symbols as Fig. 1(b)]. (d) Group data of the DFA exponent $\alpha$ as a function of the difference between the scaling properties in (b). (Inset) Root-mean-squared fluctuation $F$ of the detrended time series of the firing rates vs window width $w$, across different levels of spiking variability [symbols as in (c)]. (e) Group data of scaling relation [Eq. (4)] across the variability spectrum for freely moving mice $(n=14, N B=10$ and $\Delta T=25 \mathrm{~ms})$. Lines as in (b), with $\langle\mathrm{CV}\rangle_{*}=1.1 \pm 0.2$. (f) Scaling relation across the variability spectrum for one anesthetized monkey. Lines as in (b). (g) Linear relation between the size and lifetime critical exponents across animals. We also plot other experimental results from the ex vivo visual cortex of the turtle [28], cortical slices of the rat brain [25], freely moving mice [23], and one anesthetized monkey [22], as well as those obtained by a model [15,29]. Lower (upper) hatched regions represent the values (within standard deviations) of $\left(\tau, \tau_{t}\right)$ at $\langle\mathrm{CV}\rangle_{*}$ for the urethaneanesthetized rats (freely moving mice). 
right sides of the equation occurs around the same $\langle\mathrm{CV}\rangle$ value [Fig. 2(a)]. Results are robust with respect to changes in the timescale used to estimate firing rates and the number of $10 \mathrm{~s}$ blocks defining the resolution of the $\mathrm{CV}$ percentiles (Figs. S9-S11 [15]).

Averaging over animals [Fig. 2(b)], we obtain a critical value of spiking variability $\langle\mathrm{CV}\rangle_{*}=1.4 \pm 0.2$, therefore, far from the extremes of the variability spectrum. Moreover, when we compute the residence time distribution along this spectrum, we observe that the system spends most of the time close to the critical region [Fig. 2(b)]. This is consistent with a scenario in which the urethanized brain hovers around a critical point [31]. We found similar results in a different strain (nonalbino rats, Long Evans, $n=3$ ), using a 20\% lower spatial resolution ( 8 sites per shank) (see the Supplemental Material and Figs. S7 and S12 [15]).

Another feature of systems at the critical point is selfaffinity of time series over different timescales, as assessed by detrended fluctuation analysis (DFA) [14,32]. For our data, the root-mean-squared fluctuation $F$ scales with the length of the time window $w$ as $F \sim w^{\alpha}$ (see Supplemental Material [15]), and $\alpha$ also depends on $\langle\mathrm{CV}\rangle$ [Figs. 2(d) (inset) and S13 [15] ]. There is a remarkable convergence between these two independent signatures of criticality. We found $\alpha \approx 1$, indicating long-range time correlations and $1 / f$ noise, at the same critical value of $\langle\mathrm{CV}\rangle$ that was independently inferred by the scaling relation in Eq. (4) [Fig. 2(d)].

If the dynamics of the sensory cortex indeed hovers around a critical point, then what are the avalanche exponents at criticality? At the critical value $\langle\mathrm{CV}\rangle_{*}$, we obtained the exponents $\tau=1.52 \pm 0.09$ and $\tau_{t}=1.7 \pm 0.1$ for the anesthetized rats [Figs. S14(a) and S14(b) [15] ]. Note that, while the value of $\tau$ coincides with the critical exponent of MF-DP [33] for the rat preparation, the value of $\tau_{t}$ does not. The disagreement with the MF-DP universality class, regardless of the $\langle\mathrm{CV}\rangle$ value, is clearly seen when results are parametrically plotted in the $\left(\tau, \tau_{t}\right)$ plane [Fig. 2(g)]. We find, moreover, that other results in the literature (from diverse experimental recordings such as the ex vivo visual cortex of the turtle [28] and in vitro cultured slices of the rat cortex [25]) lie close to the linear spread of the avalanche exponents of our data [Fig. 2(g)].

Such a coincidence suggests a common underlying mechanism. A similar linear trend is found in the critical oscillations (CROS) [34] model with excitatory and inhibitory neurons, in which a transition occurs at the onset of collective oscillations, as recently described in Ref. [29] (see Supplemental Material [15] for details). A transition region in parameter space was found, where different signatures of scale-free dynamics emerge concomitantly with a peak in the power spectrum, namely, size and lifetime of avalanches are best fit by (truncated) power laws, and DFA exponents are larger than 0.5 [29]. When tuned within that transition region, the model successfully mimics our experimental results, yielding a linear spread of continuously varying exponents in the $\left(\tau, \tau_{t}\right)$ plane with a nontrivial slope [29] [Fig. 2(g)]. This supports a scenario in which the transition governing brain dynamics is not between absorbing and active phases, but rather between active and oscillating phases [34-36]. In the $(\alpha, \tau)$ and $\left(\alpha, \tau_{t}\right)$ planes, the CROS model [29] also approaches our experimental results [Figs. S14(c) and S14(d) [15] ].

To address the generality of our results, we extended our analysis to an independent sample of publicly available spiking data from freely moving mice [23]. The rationale for this investigation was to assess whether the many signatures of criticality detected under anesthesia would also be observed in nonanesthetized animals. Such parallelism, if true, would greatly extend the biological significance of the phenomena. Figure 2(e) with group results ( $n=14)$ shows that the scaling relation of Eq. (4) is indeed observed also in this more naturalistic setting [animal to animal results are detailed in Fig. S9(a) [15] ]. Note that the range of $\langle\mathrm{CV}\rangle$ values is shifted to smaller values for the mice and so is the critical value $\langle\mathrm{CV}\rangle_{*}=1.1 \pm 0.2$. At the critical point, we obtained $\tau=1.66 \pm 0.07$ and $\tau_{t}=1.9 \pm 0.1$. Despite these differences, the spread of exponents in the $\left(\tau, \tau_{t}\right)$ plane falls into the same linear relation of the anesthetized rat data [Fig. 2(g)].

To further check the robustness of our results across animal species, beyond rodents and irrespective of the anesthetic employed, we also analyzed a short ( 30 min) spiking time series of one sufentanil-anesthetized macaque monkey [22]. Despite the smaller number of points in the $\langle\mathrm{CV}\rangle$ axis, the scaling relation of Eq. (4) was also observed, with $\tau=1.77 \pm 0.005$ and $\tau_{t}=1.99 \pm 0.007$ at the critical point [Fig. 2(f)]. These values are also consistent with the other experimental results in the $\left(\tau, \tau_{t}\right)$ plane.

In conclusion, we found consistent markers of criticality in the spiking activity of the mammalian primary visual cortex, in both anesthetized (rats and monkey) and freely moving animals (mice). The critical point is neither at the synchronous nor the asynchronous ends of the spectrum, but rather at an intermediate value $\langle\mathrm{CV}\rangle_{*}$ of the coefficient of variation. Those results hold for group data and across different experimental conditions.

Importantly, our results are incompatible with a directedpercolationlike phase transition between a quiescent and an active state, a paradigm which has been a de facto theoretical workhorse of the field for over a decade [13]. We found a linear relationship between $\tau$ and $\tau_{t}$ across cortical states that encompasses results from other experimental setups and is reproduced by the CROS model.

These results open new theoretical as well as experimental avenues. On the one hand, they can guide further development of models for criticality in the brain: the CROS model does not yet provide a critical point [29] nor can it account for the differences between anesthetized and nonanesthetized results. What is the minimal model that 
can reproduce these results? On the other hand, the underlying mechanisms (neuromodulatory and other) that tune the cortex to criticality remain to be investigated. Since the critical point and the exponents change from urethanized to nonanesthetized brains, could it also be a biomarker of diseases? Moreover, it would be interesting to reanalyze our data to check how other quantifiers for cortical dynamics change with $\mathrm{CV}$ and relate to the phase transition we have found, such as the branching ratio (with the full set of techniques to account for subsampling issues [37,38]) or Kuramoto-like order parameters to assess synchrony [39].

The present results highlight the relevance of intermediate levels of spiking variability for state-dependent processing in the primary sensory cortex. We propose that, if the cortex demands both extreme modes of operation (synchronized and desynchronized) for different functions [40], it might be advantageous to self-organize near and hover over the critical point between them.

We thank F. Cysneiros for fruitful discussions and G. Buzsáki, Y. Senzai, and P. Petersen for prompt support with the freely moving mice data. This work was partially supported by the high-performance computing facilities of NPAD/UFRN. A. J. F., N. A. P. V., T. F., L. A. A. A, L. D. P., S. R., P. V.C., and M.C. acknowledge support from CAPES (Grants No. 88887.131435/2016-00 and PROEX 534/2018, No. 23038.003382/2018-39), FACEPE (Grant No. APQ 0826-1.05/15) and CNPq (Grants No. 310712/2014-9, No. 301744/2018-1, No. 425329/2018-6, No. 308775/2015-5, No. 249991/ 2013-6, and No. 408145/2016-1). This article was produced as part of the activities of FAPESP Research, Innovation and Dissemination Center for Neuromathematics (Grant No. 2013/07699-0, S. Paulo Research Foundation). C.S.-C. and B.C. acknowledge support from FCT (Grants No. SFRH/BD/51992/2012 and No. SFRH/BD/98675/2013) and PACMEDPERSYST Project No. POCI-01-0145-FEDER016428 (Portugal 2020). A. J. R. received support from an FCT Investigator Fellowship (IF/00883/2013) and acknowledges the Janssen Neuroscience Prize (first edition) and the BIAL Foundation Grant No. 30/2016.

*A. J. F. and N. A. P. V. contributed equally to this work. †pedro.carelli@ufpe.br *mcopelli@df.ufpe.br

[1] G. Moruzzi and H. W. Magoun, Electroencephalogr. Clin. Neurophysiol. 1, 455 (1949).

[2] G. Buzsaki, R. G. Bickford, G. Ponomareff, L. J. Thal, R. Mandel, and F. H. Gage, J. Neurosci. 8, 4007 (1988).

[3] R. Metherate, C. L. Cox, and J. H. Ashe, J. Neurosci. 12, 4701 (1992).

[4] E. A. Clement, A. Richard, M. Thwaites, J. Ailon, S. Peters, and C. T. Dickson, PLoS One 3, e2004 (2008).
[5] K. D. Harris and A. Thiele, Nat. Rev. Neurosci. 12, 509 (2011).

[6] A. Renart, J. de la Rocha, P. Bartho, L. Hollender, N. Parga, A. Reyes, and K. D. Harris, Science 327, 587 (2010).

[7] M. L. Schölvinck, A. B. Saleem, A. Benucci, K. D. Harris, and M. Carandini, J. Neurosci. 35, 170 (2015).

[8] N. A. P. de Vasconcelos, C. Soares-Cunha, A. J. Rodrigues, S. Ribeiro, and N. Sousa, Sci. Rep. 7, 46077 (2017).

[9] T. L. Ribeiro, M. Copelli, F. Caixeta, H. Belchior, D. R. Chialvo, M. A. L. Nicolelis, and S. Ribeiro, PLoS One 5, e14129 (2010).

[10] G. Hahn, A. Ponce-Alvarez, C. Monier, G. Benvenuti, A. Kumar, F. Chavane, G. Deco, and Y. Frégnac, PLoS Comput. Biol. 13, e1005543 (2017).

[11] D. R. Chialvo, Nat. Phys. 6, 744 (2010).

[12] J. M. Beggs and D. Plenz, J. Neurosci. 23, 11167 (2003).

[13] Criticality in Neural Systems, edited by D. Plenz and E. Niebur (Wiley, Weinheim, 2014).

[14] K. Linkenkaer-Hansen, V. V. Nikouline, J. M. Palva, and R. J. Ilmoniemi, J. Neurosci. 21, 1370 (2001).

[15] See Supplemental Material at http://link.aps.org/ supplemental/10.1103/PhysRevLett.122.208101 for a detailed description of experiments, datasets, data analysis, and model. It includes Refs. [16-21].

[16] G. Paxinos, The Rat Nervous System (Elsevier Academic Press, San Diego 2004).

[17] S. N. Kadir, D. F. M. Goodman, and K. D. Harris, Neural Comput. 26, 2379 (2014).

[18] C. Rossant, S. N. Kadir, D. F. M. Goodman, J. Schulman, M. L. D. Hunter, A. Saleem, A. Grosmark, M. Belluscio, G. Denfield, A. Ecker, A. Tolias, S. Solomon, G. Buzsáki, M. Carandini, and K. D. Harris, Nat. Neurosci. 19, 634 (2016).

[19] A. Deluca and Á. Corral, Acta Geophys. 61, 1351 (2013).

[20] S. Yu, A. Klaus, H. Yang, and D. Plenz, PLoS One 9, e99761 (2014).

[21] H. Akaike, IEEE Trans. Autom. Control 19, 716 (1974).

[22] A. Kohn and M. A. Smith, http://dx.doi.org/10.6080/ K0NC5Z4X (2016).

[23] Y. Senzai, A. Fernandez-Ruiz, and G. Buzsáki, Neuron 101, 500 (2019).

[24] G. Mochol, A. Hermoso-Mendizabal, S. Sakata, K. D. Harris, and J. de la Rocha, Proc. Natl. Acad. Sci. U.S.A. 112, 3529 (2015).

[25] N. Friedman, S. Ito, B. A. W. Brinkman, M. Shimono, R. E. Lee DeVille, K. A. Dahmen, J. M. Beggs, and T. C. Butler, Phys. Rev. Lett. 108, 208102 (2012).

[26] J. P. Sethna, K. A. Dahmen, and C. R. Myers, Nature (London) 410, 242 (2001).

[27] N. Marshall, N. M. Timme, N. Bennett, M. Ripp, E. Lautzenhiser, and J. M. Beggs, Front. Physiol. 7, 250 (2016).

[28] W. L. Shew, W. P. Clawson, J. Pobst, Y. Karimipanah, N. C. Wright, and R. Wessel, Nat. Phys. 11, 659 (2015).

[29] L. Dalla Porta and M. Copelli, PLoS Comput. Biol. 15, e1006924 (2019).

[30] J. Touboul and A. Destexhe, Phys. Rev. E 95, 012413 (2017)

[31] E. Tagliazucchi, P. Balenzuela, D. Fraiman, and D. R. Chialvo, Frontiers of Oral Physiology 3, 15 (2012). 
[32] C. Peng, S. Havlin, H. E. Stanley, and A. L. Goldberger, Chaos 5, 82 (1995).

[33] M. A. Muñoz, R. Dickman, A. Vespignani, and S. Zapperi, Phys. Rev. E 59, 6175 (1999).

[34] S.-S. Poil, R. Hardstone, H. D. Mansvelder, and K. Linkenkaer-Hansen, J. Neurosci. 32, 9817 (2012).

[35] S. di Santo, P. Villegas, R. Burioni, and M. A. Muñoz, Proc. Natl. Acad. Sci. U.S.A. 115, E1356 (2018).

[36] H. Yang, W. L. Shew, R. Roy, and D. Plenz, J. Neurosci. 32, 1061 (2012).
[37] J. Wilting and V. Priesemann, Nat. Commun. 9, 2325 (2018).

[38] J. Wilting and V. Priesemann, Cereb. Cortex, https://doi.org/ 10.1093/cercor/bhz049 (2019).

[39] A. Pikovsky, M. Rosenblum, and J. Kurths, Synchronization: A Universal Concept in Nonlinear Sciences (Cambridge University Press, Cambridge, England, 2001).

[40] M.E. Hasselmo, Behavioural Brain Research 67, 1 (1995). 